\title{
Pflanzliche Wundsignalübertragung
}

\section{Vorsicht, Verwundung! Reizweiterlei- tung mit mechanosensitiven Proteinen}

MICHAEL M. WUDICK

INSTITUT FÜR MOLEKULARE PHYSIOLOGIE, UNIVERSITÄT DÜSSELDORF

\section{Being sessile, plants are exposed to adverse stresses, including wounding by insects. Albeit lacking experimental evidence, one hypothesis predicted involvement of hydro-electric signals in wound signaling. Now, we could show that the mechanosensitive anion channel MSL10 is necessary for wound-induced long-distance signal- ing in plants. By linking mechano-sensing, ion fluxes, membrane depo- larization and electrical signal propagation, MSL10 might integrate hydraulic and electric wound signals.}

DOI: $10.1007 / \mathrm{s} 12268-021-1658-5$

(C) Der Autor 2021

Pflanzen sind im Laufe ihres Lebenszyklus einer Vielzahl äußerer Faktoren ausgesetzt, wie Klimaschwankungen oder dem Befall von Insekten und Schädlingen. Schätzungsweise gehen weltweit jährlich bis zu 20 Prozent der Ernteerträge durch Schädlingsbefall verloren [1]. Ein besseres Verständnis über pflanzliche Abwehrstrategien gegen Insektenfraß ist deshalb nicht nur aus ökonomischer Sicht, sondern auch mit Blick auf eine gesicherte Lebensmittelversorgung hoch relevant.

Pflanzliche Abwehrstrategien: vom mechanischen Schutzschild bis zur „chemischen Keule“

Zur Abwehr von Fressfeinden wie Insekten haben Pflanzen verschiedene Strategien entwickelt. Als erster Schutz dienen oft äußere Barrieren, z. B. in Form von Borke, Stacheln oder wachsbeschichteten Blättern. Wird diese Barriere durchbrochen, kann die Pflanze durch gezielte Hemmstoffproduktion verhindern, dass Schädlinge sich auf andere Pflanzenteile oder Nachbarpflanzen ausbreiten. Verwundung führt zur Produktion des pflanzlichen Hormons Jasmonsäure, das die Freisetzung von Abwehrstoffen und somit die Immunabwehr der Pflanze ankurbelt. Jasmonsäure kann dabei in verschiedenen chemischen Varianten auftreten. In Form des gasförmigen Methyljasmonats kann es über Spaltöffnungen im Blatt ausströmen und Nachbarpflanzen vor anstehendem Befall durch Fressfeinde „warnen“ [2]. Zudem kann Jasmonsäure die Produktion von toxischen Substanzen veranlassen. Wenn Pflanzen beispielsweise von Raupen angefressen werden, produzieren sie bestimmte Hemmstoffe, die die Verdauungsenzyme der Raupen blockie- ren. Dadurch wird die Nahrungsaufnahme und das Wachstum der Raupen beeinträchtigt und die Pflanze als Nahrungsquelle unattraktiver [3]. Damit diese Abwehrstoffe nicht nur im verwundeten Blatt, sondern in der ganzen Pflanze hergestellt werden, sendet die Pflanze Signale bis in unverwundete Gewebeteile. Diese wundinduzierte Signalweiterleitung kann dabei auf unterschiedliche Weise erfolgen.

Signalweiterleitung in Pflanzen: elektrisch, chemisch oder hydraulisch?

Die Ausbreitung des Wundsignals erfolgt über das Leitgewebe der Pflanze, das den Transport von Wasser und darin gelöster Nährstoffe ermöglicht. Die Weiterleitung kann in Form elektrischer, chemischer oder hydraulischer Signale erfolgen, oder einer Kombination daraus. Die Blätter der Modellpflanze Arabidopsis thaliana (Ackerschmalwand) wachsen in Rosettenform und sind durch ihr sequenzielles Wachstum von „alt“ nach ,jung“ klassifizierbar (Abb. 1). Zudem kann man anhand der Blattstellung identifizieren, welche Blätter über dasselbe Leit-

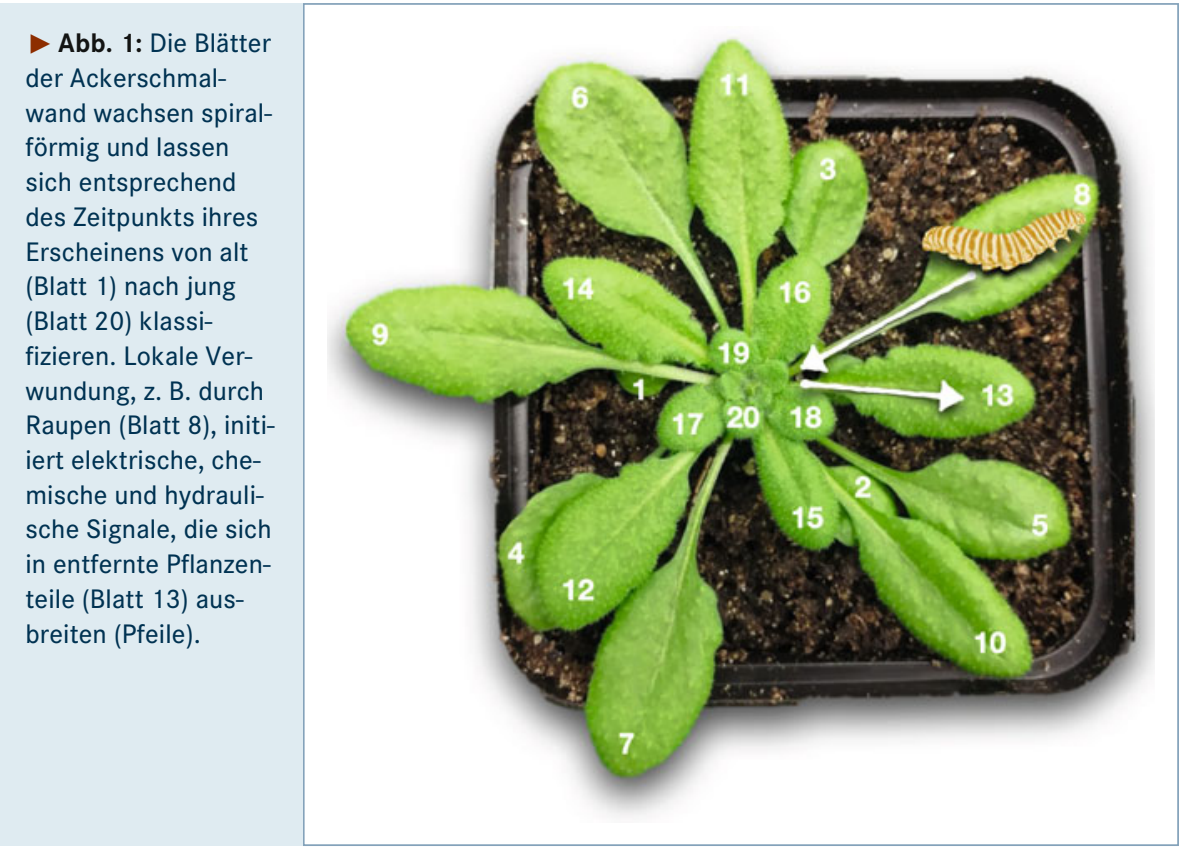



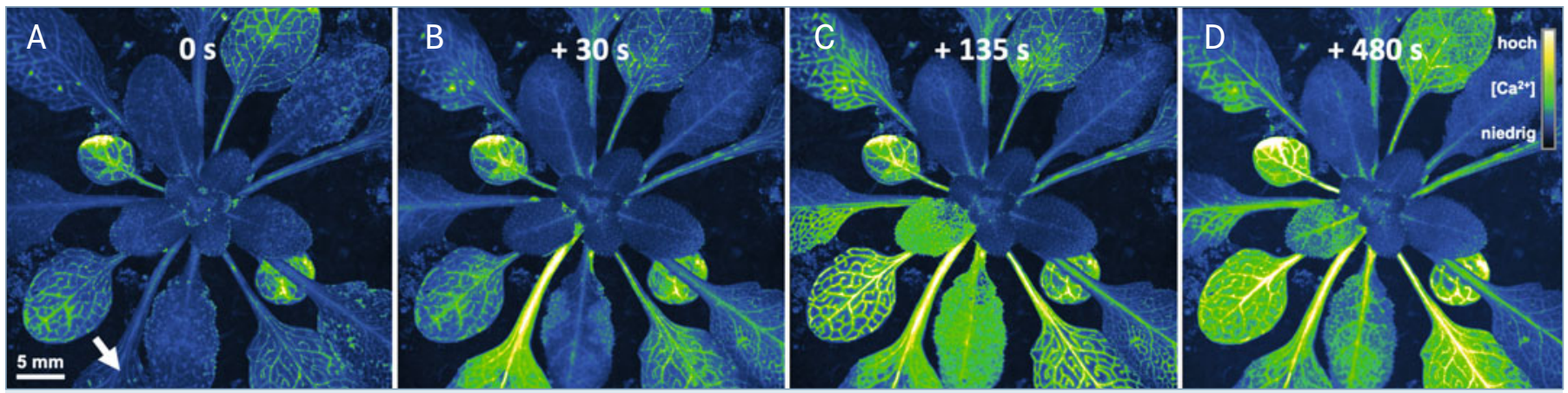

$\triangle$ Abb. 2: Visualisierung wundinduzierter Calciumsignale durch den fluoreszierenden Calciumsensor MCaMP6s. Mechanische Blattverwundung (A, Pfeil) simuliert Insektenfraß und führt zum lokalen Anstieg der Calciumkonzentration $\left[\mathrm{Ca}^{2+}\right]$ im Leitgewebe (B). Das Signal gelangt dann in benachbarte, über gemeinsame Leitgewebe verbundene Blätter (C), bevor es sich auf die restliche Pflanze ausbreitet (D). ( ) Bild: C. Sies, HHU.

\section{Kontrollpflanze}

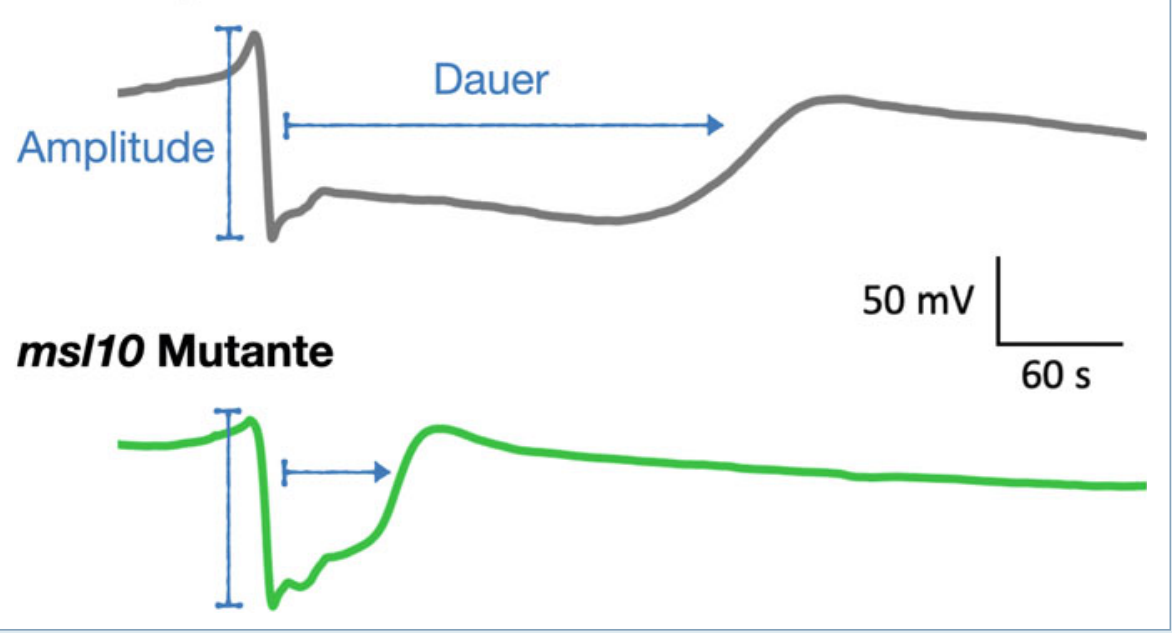

A Abb. 3: Repräsentative Spannungskurven von Kontrollpflanzen (grau) und ms/10-Mutanten (grün). Im abgebildeten Beispiel sind sowohl die Dauer (in Sekunden, s) als auch die Amplitude (in Millivolt, $\mathrm{mV}$ ) des elektrischen Signals in der ms/10-Mutante verändert, was z. B. zu einer verminderten Produktion von Abwehrstoffen führen kann.

gewebe zum direkten Signalaustausch befähigt sind. Durch den Fressfeind oder die Verwundung freigesetzte Botenstoffe können an spezielle Rezeptoren im Leitgewebe binden. Einige dieser Rezeptoren fungieren als Kanäle, die den Transport von Ionen über biologische Membranen ermöglichen. Ionenkanäle können das chemische Wundsignal in ein elektrisches Signal umwandeln, verstärken und an Nachbarzellen übermitteln. Glutamat ist eines der am besten erforschten wundinduzierten Signalmoleküle. Ähnlich wie im menschlichen Gehirn, wo Glutamat als Neurotransmitter die neuronale Zell-Zell-Kommunikation ermöglicht, verfügt das Leitgewebe von Pflanzen über Glutamatrezeptoren (GLRs), an die der Botenstoff binden kann [4]. Die Interaktion mit Glutamat erhöht die Calcium $\left(\mathrm{Ca}^{2+}\right)$-Transportaktivität der GLRs und führt zur Glutamat-abhängigen Ausbreitung des $\mathrm{Ca}^{2+}$-Signals $[5,6]$.

\section{Visualisierung wundinduzierter Signalmoleküle}

Genetisch codierte, fluoreszenzbasierte Sensoren für Glutamat und $\mathrm{Ca}^{2+}$-Ionen erlauben es, die Entstehung und Ausbreitung des Wundsignals in Echtzeit quantitativ zu analysieren (Abb. 2). Vorangegangene Experimente zeigten, dass bestimmte GLRs essenziell für die Ausbreitung wundinduzierter $\mathrm{Ca}^{2+}$ - und elektrischer Signale über das Leitgewebe sind [7]. Über die Bedeutung des Leitgewebes für die Ausbreitung eines hydraulischen Wundsignals und die Kopplung hydroelektrischer Signale wurde lange spekuliert [8, 9]. Im Leitgewebe herrscht durch den kontinuierlichen Transpirationssog zwischen der Wurzel und oberirdischen
Pflanzenteilen ein Unterdruck. Mechanistische Modelle nahmen an, dass die Verwundung des Leitgewebes zu einem rapiden Druckanstieg in den Leitgewebezellen führt. Die sich ausbreitende Druckdifferenz könnte von mechanosensitiven Proteinen erfasst und in elektrische Signale umgewandelt werden. Daran beteiligte Proteine waren bislang jedoch unbekannt.

\section{Das mechanosensitive Protein MSL10 ist essenziell bei der Wundsignalübertragung}

Elektrische Signale lassen sich in Blättern als Oberflächenpotenziale - die surface oder slow wave potentials (SWPs) - detektieren. Die Messung von SWPs erfolgt mithilfe von Elektroden, die auf Blattstielen von verwundeten und unversehrten Blättern platziert werden und die gesamte elektrische Aktivität des darunterliegenden Gewebes erfassen. Basierend auf diesen Informationen haben wir ein Messverfahren optimiert, bei dem nach mechanischer Verwundung von Blatt 8 das dabei generierte elektrische Wundsignal in Blatt 13 gemessen wird (Abb. 1). Kontrollpflanzen sind durch SWPs mit charakteristischer Dauer und Amplitude gekennzeichnet (Abb. 3, [6, 7]). Anhand systematischer Screens von Mutanten, denen bestimmte Gene fehlten, entdeckten wir, dass Pflanzen ohne den mechanosensitiven Anionenkanal MSL10 signifikant verkürzte SWPs aufwiesen [10]. Zudem kam es in diesen Pflanzen zu einer Verringerung des wundinduzierten $\mathrm{Ca}^{2+}$-Signals und einer verminderten Produktion von Abwehrstoffen [10]. Die Ausbreitung des Botenstoffs Glutamat war hingegen nicht beeinträchtigt. Da das gleichzeitige Entfernen der ebenfalls zur Wundsignalübertragung benötigten GLRs zu keiner weiteren Veränderung des SWPs führte, ist unsere Hypothese, dass entweder zwei parallele Sig- 
nalwege existieren oder dass sich MSL10 und die GLRs in einem gemeinsamen Signalweg gegenseitig regulieren können [10].

\section{Glutamat als Wundsignal oder Nährstoff: Wie unterscheidet die Pflanze?}

Anders als im tierischen System fungiert Glutamat in Pflanzen nicht nur als Botenstoff, sondern auch als Metabolit, dessen Konzentration ständigen Schwankungen unterliegt. Um zwischen metabolischen Schwankungen und verwundungsbedingtem Anstieg der Glutamatkonzentration $\mathrm{zu}$ unterscheiden, bedarf es eventuell einer weiteren Stellschraube. Diese Funktion übernimmt möglicherweise MSL10. In unserem Modell führt wundinduzierter Druckabfall im Blatt-Leitgewebe zur MSL10-Aktivierung und dem Transport von Anionen über die Zellmembran. Die so hervorgerufene Membrandepolarisierung könnte der Pflanze helfen, den gleichzeitigen Anstieg der Glutamatkonzentration als Wundsignal zu erkennen und GLR-vermittelte Abwehrreaktionen einzuleiten (z. B. durch Jasmonsäure-Produktion). In der Tat existiert eine ähnliche Form der simultanen Ko-Aktivierung bei einigen tierischen Glutamatrezeptoren, bei denen die Interaktion der Rezeptoren mit Glutamat nur bei gleichzeitiger Membrandepolarisierung zu einer Signalweiterleitung führt [11]. Indem wir die an der pflanzlichen Wundsignalweiterleitung beteiligten Prozesse und Komponenten besser verstehen, können wir den Pflanzen möglicherweise helfen, Bedrohungen schneller zu erkennen und abzuwehren. Zukünftig kann so eventuell Ernteausfällen durch Schädlingsbefall gezielter entgegengewirkt werden.

\section{Danksagung}

Dieses Projekt wurde durch die Deutsche Forschungsgemeinschaft (DFG) - Projekt- nummer 267205415 - SFB 1208 gefördert und ich danke allen daran Beteiligten!

\section{Literatur}

[1] Deutsch CA, Tewksbury JJ, Tigchelaar M et al. (2018) Increase in crop losses to insect pests in a warming climate. Science 361: 916-919

[2] Farmer EE, Ryan CA (1990) Interplant communication: airborne methyl jasmonate induces synthesis of proteinase inhibitors in plant leaves. Proc Natl Acad Sci USA 87: 7713-7716

[3] Green TR, Ryan CA (1972) Wound-induced proteinase inhibitor in plant leaves: a possible defense mechanism against insects. Science 175: 776-777

[4] Alfieri A, Doccula FG, Pederzoli R et al. (2020) The structural bases for agonist diversity in an Arabidopsis thaliana glutamate receptor-like channel. Proc Natl Acad Sci USA 117: 752-760

[5] Wudick MM, Portes MT, Michard E et al. (2018) CORNICHON sorting and regulation of GLR channels underlie pollen tube $\mathrm{Ca}^{2}+$ homeostasis. Science 360 : 533-536

[6] Toyota M, Spencer D, Sawai-Toyota S et al. (2018) Glutamate triggers long-distance, calcium-based plant defense signaling. Science 361: 1112-1115

[7] Mousavi SAR, Chauvin A, Pascaud F et al. (2013) GLUTAMATE RECEPTOR-LIKE genes mediate leaf-to-leaf wound signalling. Nature 500: 422-426

[8] Malone M (1996) Rapid, long-distance signal transmission in higher plants. Adv Bot Res 22: 163-228 [9] Farmer EE, Gasperini D, Acosta IF (2014) The squeeze cell hypothesis for the activation of jasmonate synthesis in response to wounding. New Phytol 204: 282-288

[10] Moe-Lange J, Gappel NM, Machado M et al. (2021) Interdependence of a mechanosensitive anion channel and glutamate receptors in distal wound signaling. Sci Adv 7: eabg4298

[11] Hansen KB, Yi F, Perszyk RE et al. (2018) Structure, function, and allosteric modulation of NMDA receptors. J Gen Physiol 150: 1081-1105

Funding
DEAL.
Open Ace

unding note: Open Access funding enabled and organized by Projekt

Open Access: Dieser Artikel wird unter der Creative Commons
Namensnennung 4.0 International Lizenz veröffentlicht, welche die Nutzung, Vervielfältigung, Bearbeitung, Verbreitung und Wiedergabe in jeglichem Medium und Format erlaubt, sofern Sie den/die ursprünglichen Autor(en) und die Quelle ordnungsgemäß nennen, einen Link zur Creative commons Lizenz beifugen und angeben, ob Anderungen vorgenommen wurden. Die in diesem Artikel enthaltenen Bilder und sonstiges Drittmaterial unterliegen ebenfalls der genannten Creative Commons Lizenz, sofern sich aus der Abbildungslegende nichts anderes ergib. Sofern das betreffende Material nicht unter der genannten Creative gesetzlichen Vorschriften erlaubt ist, ist für die oben aufgeführten Weiterverwendungen des Materials die Einwilligung des jeweiligen Weiterverwendungen des Materials die Einwilligung des jeweiligen
Rechteinhabers einzuholen. Weitere Details zur Lizenz entnehmen Sie bitte der Lizenzinformation auf http://creativecommons.org/licenses/by/4.0/ deed.de.

\section{Korrespondenzadresse:}

Dr. Michael M. Wudick

Institut für molekulare Physiologie

Heinrich-Heine-Universität Düsseldorf

Universitätsstraße 1

D-40225 Düsseldorf

wudick@hhu.de

\section{Hier steht eine Anzeige.}

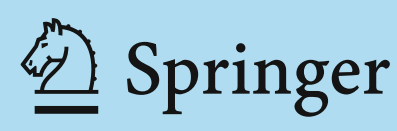

